\title{
Superlattice structures observed in the extraordinary phase sequence and analyzed by the phenomenological Landau model and the partially molecular model
}

\author{
K. L. Sandhya, ${ }^{1, *}$ A. D. L. Chandani, ${ }^{1,2}$ Atsuo Fukuda, ${ }^{1, \dagger}$ S. Kumar, ${ }^{1,3}$ and Jagdish K. Vij ${ }^{1,4, \ddagger}$ \\ ${ }^{1}$ Department of Electronic and Electrical Engineering, Trinity College, University of Dublin, Dublin 2, Ireland \\ ${ }^{2}$ Department of Chemistry, Faculty of Science, University of Peradenia, Peradenia, Sri Lanka \\ ${ }^{3}$ Raman Research Institute, Bangalore, India \\ ${ }^{4}$ School of Electronic and Electrical Engineering, Sungkyunkwan University, Suwon 440-476, Korea
}

(Received 8 April 2013; published 19 June 2013)

\begin{abstract}
We draw several electric-field-temperature $(E-T)$ phase diagrams with electric-field-induced birefringence contours in the $n$ OHFBBB1M7 $(n=10)$ and $n$ OTBBB1M7 $(n=11)(\mathrm{C} 11)$ mixture system by changing the C11 concentration carefully; some of the mixtures show the unusual extraordinary phase sequence where subphases with the four-, five-, and six-layer superlattice structures emerge above the smectic- $C^{*}$ main phase. We try to understand the results in terms of two complementary models that have so far been proposed: the phenomenological Landau model of phase transitions by Dolganov et al. [P. V. Dolganov et al., Phys. Rev. E 86, 020701(R) (2012)] and the partially molecular Emelyanenko-Osipov model [A. V. Emelyanenko and M. A. Osipov, Phys. Rev. E 68, 051703 (2003)]. The observed E-T phase diagram can be well reproduced by the phenomenological model. An emergence of the subphase with the four-layer superlattice structure above smectic- $C^{*}$ is also understandable in terms of the partially molecular model. We discuss the pros and cons of the two models as well.
\end{abstract}

DOI: 10.1103/PhysRevE.87.062506

PACS number(s): 61.30.Eb, 64.70.M-, 77.80.-e

\section{INTRODUCTION}

The phase transition between chiral tilted smectic liquid crystals $\mathrm{Sm}-C_{A}^{*}$ and $\mathrm{Sm}-C^{*}$ is usually of first order and the system is frustrated between the synclinic ferroelectric and anticlinic antiferroelectric orderings. The frustration together with the long-range interlayer interactions (LRILIs) may cause the temperature-induced sequence of phase transitions [1-3], producing a variety of optically biaxial polar subphases between the two main phases $\mathrm{Sm}-C_{A}^{*}$ and $\mathrm{Sm}-C^{*}$ [4-12]. Biaxial subphases have nonplanar superlattice structures with highly distorted microscopic short-pitch helical director arrangements in their unit cells [13-22]. We have insisted on the appropriateness of specifying the biaxial subphases, which emerge sequentially in the temperature-induced transition, by the relative ratio of ferroelectric $[F]$ and antiferroelectric $[A]$ orderings in the superlattice structure unit cell, such as Sm$C_{A}^{*}\left(q_{T}\right)$, where $q_{T}=[F] /([A]+[F])$. The subphases with three- and four-layer superlattice structures $\mathrm{Sm}-C_{A}^{*}\left(\frac{1}{3}\right)$ and $\mathrm{Sm}-C_{A}^{*}\left(\frac{1}{2}\right)$ were well established by resonant $\mathrm{x}$-ray scattering (RXRS). Additional subphases $\mathrm{Sm}-C_{A}^{*}\left(\frac{1}{4}\right), \mathrm{Sm}-C_{A}^{*}\left(\frac{2}{5}\right)$, and $\mathrm{Sm}-C_{A}^{*}\left(\frac{3}{5}\right)$ have been reported to exist by performing careful electro-optical investigations [7-11]. Near the transition temperature to $\mathrm{Sm}-A$, the frustration may occur among the three main phases $\mathrm{Sm}-C_{A}^{*}, \mathrm{Sm}-C^{*}$, and $\mathrm{Sm}-A$ [6]. The optically uniaxial polar $\mathrm{Sm}-C_{\alpha}^{*}$ subphase may also emerge directly below Sm- $A$ and replace Sm- $C^{*}$ in some cases [23-25]. It has been firmly established by RXRS and other sophisticated techniques that $\mathrm{Sm}-C_{\alpha}^{*}$ is characterized by a microscopic

\footnotetext{
*Present address: Department of Physics, M. S. Ramaiah Institute of Technology, Bangalore-54, India.

†fukudaa@tcd.ie

$¥$ jvij@tcd.ie
}

helical structure with an incommensurate short pitch ranging from a few to several tens of smectic layers [13,14,26-28]. There is no essential difference between $\mathrm{Sm}-C_{\alpha}^{*}$ and Sm- $C^{*}$, except for their helical pitch lengths.

Recently, Wang et al. [22] observed a superlattice structure with a six-layer unit cell by RXRS. With this finding, our works for the past decade have been vindicated since we have continued to insist on the emergence of subphases with unit cells larger than four smectic layers [4-12]. Soon after this observation was reported, Chandani et al. [10] studied the electric-field-induced birefringence (EFIB) of the same mixture system and drew the contours of constant birefringence on the electric-field-temperature $(E-T)$ phase diagram and showed the emergence of an additional subphase with a unit cell of five layers in the phase sequence Sm- $C^{*}-$ $\mathrm{Sm}-C_{d 4}^{*}-\mathrm{Sm}-C_{d 5}^{*}-\mathrm{Sm}-C_{d 6}^{*}-\mathrm{Sm}-C_{\alpha}^{*}-\mathrm{Sm}-A$. Here the subscript $d$ refers to a distorted microscopic helical structure and the subscript number indicates its short pitch $p_{q T}$. Since the short pitch $p_{q T}$ is uniquely determined by $q_{T}$ as $\left|p_{q T}\right|=$ $2 /\left(1-q_{T}\right)$, the phase sequence can be written in terms of $q_{T}$ as Sm- $C^{*}-\mathrm{Sm}-C^{*}\left(\frac{1}{2}\right)-\mathrm{Sm}-C^{*}\left(\frac{3}{5}\right)-\mathrm{Sm}-C^{*}\left(\frac{2}{3}\right)-\mathrm{Sm}-C_{\alpha}^{*}-$ $\mathrm{Sm}-A$. This phase emerging sequence is quite unusual and extraordinary during the long history of studies on chiral polar smectic liquid crystals [29-33]; the low-temperature main phase is not $\mathrm{Sm}-C_{A}^{*}$ but $\mathrm{Sm}-C^{*}$, so it would be better to write $\mathrm{Sm}-C^{*}\left(q_{T}\right)$, but not $\mathrm{Sm}-C_{A}^{*}\left(q_{T}\right)$. Hereafter we simply specify $q_{T}$ in designating biaxial subphases; for example, the above unusual and extraordinary phase sequence is written as Sm- $C^{*}-\frac{1}{2}-\frac{3}{5}-\frac{2}{3}-\mathrm{Sm}-C_{\alpha}^{*}-\mathrm{Sm}-A$.

Quite recently, using the phenomenological Landau theory of the phase transitions, Dolganov et al. [34] studied this unusual extraordinary sequence and showed that both phases exist at zero field and that $\frac{2}{3}$ transforms into $\frac{3}{5}$ for relatively low fields. They calculated the $E-T$ phase diagram, which can then be compared with the experimental one. Since no 
detailed investigations were performed in our previous paper [10], we carefully study EFIB in the same $n$ OHFBBB $1 \mathrm{M} 7$ $(n=10)(10 \mathrm{OHF})$ mixture system of several $n$ OTBBB1M7 $(n=11)$ (C11) concentrations and draw $E-T$ phase diagrams with contours of constant birefringence for each of the concentrations studied. The results will be presented and discussed in terms of Dolganov's phenomenological Landau model of the phase transitions. This is one of the dual purposes of this paper.

The phenomenological Landau model proposed by Dolganov et al. [34-37] is quite heuristic and can similarly analyze both ordinary and extraordinary phase sequences just by an appropriate choice of parameters. In other words, it would have been meaningless to consider one phase sequence for usual ordinary and the second as unusual extraordinary; both phase sequences are similarly possible using different parameters. In the quasimolecular model proposed by Emelyanenko and Osipov, on the contrary, it is meaningful to ask why and how the extraordinary phase sequence appears; they treated the short-range interlayer interactions (SRILIs) phenomenologically and analyzed LRILIs on the molecular basis in terms of the dipole-dipole and dipole-quadrupole interactions. The mixtures under study exhibit the biaxial subphases with multilayer unit cells above Sm- $C^{*}$. Consequently, we need to reconsider the fundamental premise that the biaxial subphases with multilayer unit cells always emerge between the main phases $\mathrm{Sm}-C_{A}^{*}$ and $\mathrm{Sm}-C^{*}$ as a consequence of the frustration between them. Before Dolganov et al. [35] reported the phenomenological model, the quasimolecular model proposed by Emelyanenko and Osipov had been the only model that was able to satisfactorily explain the staircase character of the subphases as well as their microscopic short-pitch distorted helical structures. Since both models, phenomenological and molecular, are complementary to each other and moreover since we have been using the Emelyanenko-Osipov model [3] together with our own primitive way of understanding the frustration in clinicity to explain our results, we will consider the extent to which the unusual extraordinary phase sequence can also be understood in a similar line of thought using their LRILIs. This is the second purpose of this paper.

\section{EXPERIMENT}

Samples used are the binary mixtures of $10 \mathrm{OHF}$ and C11, the structural formula of which are given in Fig. 1. The major constituent of the binary mixtures, 10OHF, shows the unusual extraordinary phase sequence $\mathrm{Sm}-C^{*}-\frac{1}{2}-\mathrm{Sm}$ -

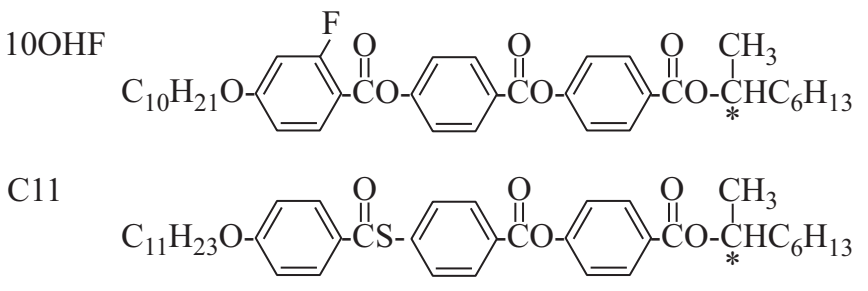

FIG. 1. Chemical structures of the compounds used in the present experiments. Both are $S$ moieties. 10OHF shows the unusual extraordinary phase sequence $\mathrm{Sm}-C^{*}-\frac{1}{2}-\mathrm{Sm}-C_{\alpha}^{*}-\mathrm{Sm}-A$, whereas $\mathrm{C} 11$ has the usual ordinary phase sequence $\mathrm{Sm}-C_{A}^{*}-\frac{1}{2}-\mathrm{Sm}-C_{\alpha}^{*}-\mathrm{Sm}-A$.
$C_{\alpha}^{*}-\mathrm{Sm}-A$, where $\mathrm{Sm}-C^{*}$ is the lowest-temperature phase. Antiferroelectric Sm- $C_{A}^{*}$ does not emerge even at low temperatures and all the subphases lie above ferroelectric Sm- $C^{*}$. Homeotropically aligned $25-\mu \mathrm{m}$-thick cells were prepared to measure EFIB using a photoelastic modulator (PEM) setup. The cell was constructed using a bottom glass plate with two indium tin oxide (ITO) electrodes separated by a distance of $180 \mu \mathrm{m}$ and a top normal glass plate set apart from the bottom one by a $25-\mu \mathrm{m}$ polyethylene terephthalate spacer. The two ITO electrodes on the bottom glass plate were used for applying an in-plane electric field for measuring EFIB. Homeotropic alignment in a cell was achieved by spin coating the inner surfaces of the glass substrates with carboxy chromium complex (chromolane) and by curing at a temperature of $150{ }^{\circ} \mathrm{C}$ for $30 \mathrm{~min}$. The cell was heated and filled with a sample mixture in the isotropic phase and cooled slowly to $\mathrm{Sm}-A$. The cell was in a temperature-controlled hot stage, where the temporal fluctuation is regulated within an accuracy of $0.01{ }^{\circ} \mathrm{C}$ or better, but the spatial uniformity throughout the aperture of $1 \mathrm{~mm} \phi$ is approximately $0.1^{\circ} \mathrm{C}$. The homeotropic alignment was confirmed by examining the cell under the polarizing microscope.

The EFIB at various temperatures was measured as a function of electric field on applying a $10-\mathrm{Hz}$ bipolar square wave of varying amplitude from $5 / 180$ to $275 / 180 \mathrm{~V} / \mu \mathrm{m}$; the applied field is increased stepwise at $2.5 / 180 \mathrm{~V} / \mu \mathrm{m}$ and stayed at each value for $9 \mathrm{~s}$ to accumulate an EFIB signal. This was done in first cooling and then heating modes. The entire temperature range was approximately between $45{ }^{\circ} \mathrm{C}$ $\left(\mathrm{Sm}-C^{*}\right)$ and $95^{\circ} \mathrm{C}(\mathrm{Sm}-A)$. The cells were not allowed to cool down to the crystalline phase in order to prevent destruction of the alignment. The homeotropic cells of these binary mixtures become crystalline around $45^{\circ} \mathrm{C}$. The temperature was changed at a step of $0.02,0.05$, or $0.1{ }^{\circ} \mathrm{C}$ and the waiting time for structure stabilization at every temperature point was $0.5 \mathrm{~min}$. The PEM used was a commercial one, PEM-90 (Hinds Instruments, Hillsboro, OR), which has a resonant frequency of $50 \mathrm{kHz}$. Detailed explanations of the PEM-based setup were given in our previous papers for measuring EFIB [6,7,38]. The $E-T$ phase diagrams were constructed by plotting EFIB contours. The contours showed characteristic patterns typical of the prototype subphases $\frac{1}{2}, \frac{1}{3}$, and $\mathrm{Sm}-C_{\alpha}^{*}$ as well as the main phases $\mathrm{Sm}-C_{A}^{*}, \mathrm{Sm}-C^{*}$, and $\mathrm{Sm}-A$, although it may not be easy to distinguish between $\frac{1}{2}$ and $\mathrm{Sm}-C_{A}^{*}$ in some cases. In the binary mixture system under investigation, since RXRS unambiguously showed the emergence of Sm- $C^{*}, \frac{1}{2}, \mathrm{Sm}-C_{\alpha}^{*}$, and $\mathrm{Sm}-A$, identifying these by EFIB was easily facilitated in our previous work [10].

The $E-T$ phase diagrams thus obtained actually depend on several experimental parameters: $\mathrm{C} 11$ concentrations, cooling and heating modes, temperature changing rates, scanned temperature ranges, the applied maximum field responsible for sample degradation, etc. It is worthwhile to review some of the parameters here, although limited experimental studies were carried out so far. The previous EFIB measurements were performed in two mixtures containing 11.05 and $11.48 \mathrm{wt} \%$ of C11 in 10OHF [10]. A ferrielectric phase identified as $\frac{3}{5}$ was undoubtedly shown to emerge in a temperature range as wide as $2{ }^{\circ} \mathrm{C}$ or more between $\frac{1}{2}$ and $\mathrm{Sm}-C_{\alpha}^{*}$, which corresponds to the noisy region in RXRS reported by Wang et al. On the 

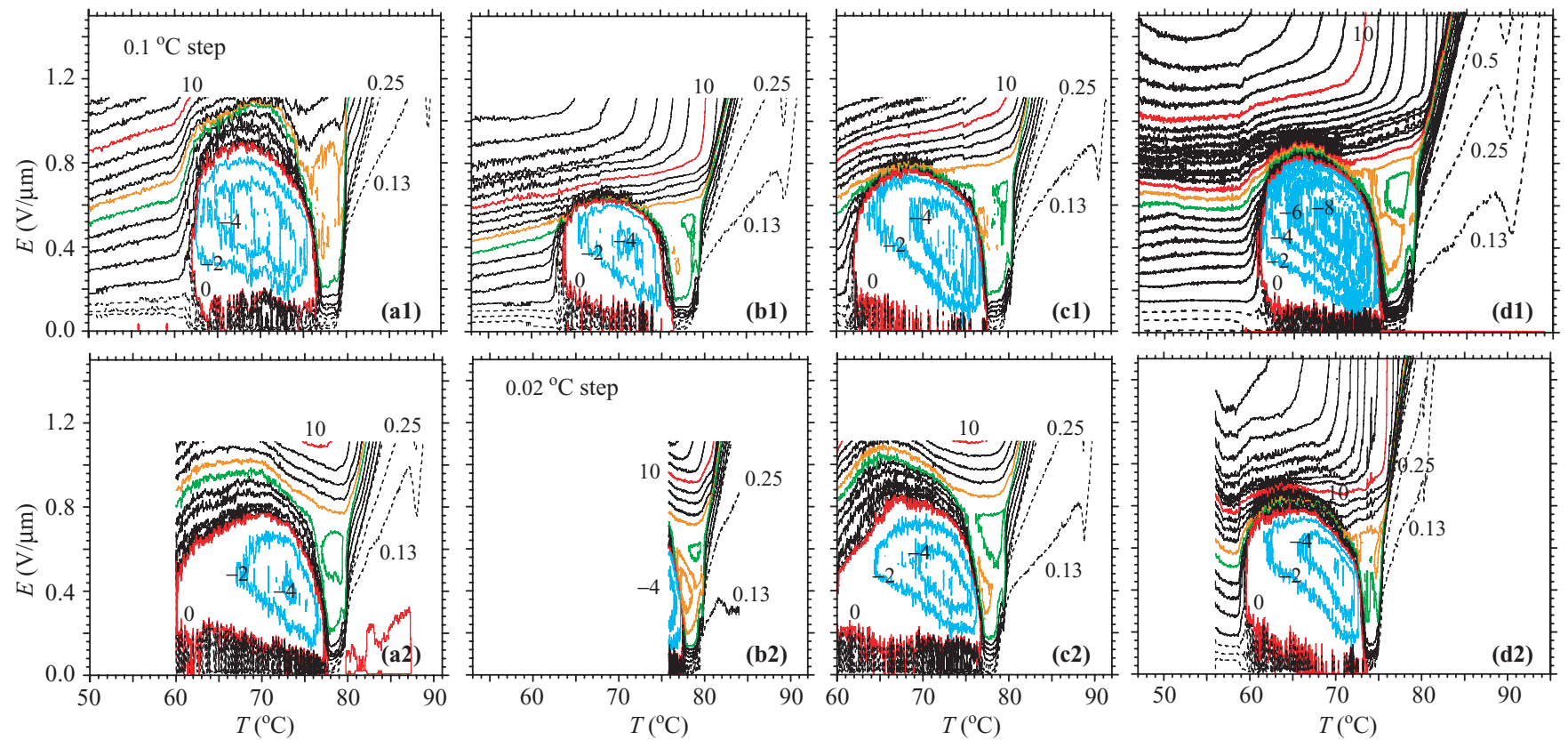

FIG. 2. (Color online) $E-T$ phase diagrams for the binary mixtures of $10 \mathrm{OHF}$ containing (a) 11.05, (b) 12.45, (c) 13.50, and (d) 15.00 wt. \% of $\mathrm{C} 11$ measured in several experimental parameter settings; the upper (1) and lower (2) phase diagrams were obtained in the cooling and heating modes, respectively, at a temperature change step of $0.05{ }^{\circ} \mathrm{C}$, except for $0.1{ }^{\circ} \mathrm{C}$ in $(\mathrm{a} 1)$ and $0.02{ }^{\circ} \mathrm{C}$ in (b2). The scanned temperature range was very narrow in (b2). Contours are drawn by solid lines at an interval of $\Delta n=1 \times 10^{-3}$, with some auxiliary contours by dashed lines in smaller steps of $\Delta n$; closed contours characteristic of $\frac{1}{2}$ with negative $\Delta n$ values are very noisy and hence drawn at an interval of $\Delta n=-2 \times 10^{-3}$. Applying $1.5 \mathrm{~V} / \mu \mathrm{m}$ caused sample degradation and a resulting decrease in the phase transition temperature between $\mathrm{Sm}-C_{\alpha}^{*}$ and $\mathrm{Sm}-A$, as seen in (d1) and (d2).

contrary, the emerging temperature range of antiferroelectric $\frac{2}{3}$ was unexpectedly narrow as compared with that observed in RXRS by Wang et al. To see the concentration dependence of this difference, four mixtures containing 11.05, 12.45, 13.50, and 15.00 wt. \% of $\mathrm{C} 11$ in $10 \mathrm{OHF}$ are studied in this paper. We wanted to use a temperature changing step of $0.02{ }^{\circ} \mathrm{C}$ so that we can detect any subphase emerging over a temperature range as narrow as $0.2{ }^{\circ} \mathrm{C}$. To make a compromise with available measuring time, however, most $E-T$ phase diagrams are obtained using a step of $0.05{ }^{\circ} \mathrm{C}$. Regarding the maximum applied electric field, we noticed that $1.2 \mathrm{~V} / \mu \mathrm{m}$ or less is desirable for avoiding sample degradation and a resulting decrease in the phase transition temperature between $\mathrm{Sm}-C_{\alpha}^{*}$ and $\mathrm{Sm}-A$ after one cycle measurement in cooling and heating. As in [10], a bipolar square-wave field is used to avoid any charge accumulation on an electrode and a frequency of $10 \mathrm{~Hz}$ is empirically chosen so that the optimum collection of EFIB signals can be performed $[6,39]$.

\section{RESULTS}

Setting aside the fine details, let us see the big picture of observed $E-T$ phase diagrams in the first instance. They are rather similar to each other, irrespective of $\mathrm{C} 11$ concentrations, cooling and heating modes, temperature changing rates, scanned temperature ranges, the maximum applied field, etc., as illustrated in Fig. 2. Considerable differences are conspicuous such as different $10 \mathrm{OHF}$ used, however, when we compare Fig. 2(a) with Fig. 3(b) of the previous paper [10]; we admit that data processing requires us to multiply the ordinate axis of Fig. 3(b) by a factor of 2. Contours show characteristic patterns in these $E-T$ phase diagrams [6-9]. We can easily identify the four phases, synclinic ferroelectric $\mathrm{Sm}-C^{*}$, four-layer antiferroelectric $\frac{1}{2}$, optically uniaxial Sm$C_{\alpha}^{*}$, and nontilted paraelectric Sm- $A$, which were confirmed to emerge by RXRS [22], as indicated in the lower part of Fig. 3(a). Dashed lines with an oblique steep rise with temperature are observed at around $90{ }^{\circ} \mathrm{C}$, which show the electroclinic effect in $\mathrm{Sm}-A$. Solid and dashed lines increasing less steeply with temperature observed below $\mathrm{Sm}-A$ represent the unwinding processes of the microscopic short-pitch helical structure of Sm- $C_{\alpha}^{*}$. The phase transition between Sm- $A$ and $\mathrm{Sm}-C_{\alpha}^{*}$ can be clearly seen as dents in the dashed lines. At higher fields and in the low-temperature region of $\mathrm{Sm}-C_{\alpha}^{*}$, contours become nearly vertical and represent unwound Sm$C^{*}$. It should be noticed that the maximum applied field of $1.5 \mathrm{~V} / \mu \mathrm{m}$ conspicuously degrades the sample quality, decreases the phase transition temperature between $\mathrm{Sm}-C_{\alpha}^{*}$ and $\mathrm{Sm}-A$, and reduces the $\mathrm{Sm}-C_{\alpha}^{*}$ temperature range after one-cycle measurement in cooling and heating, as clarified by comparing Figs. 2(d1) and 2(d2).

It has been well established that contour lines may form negative closed loops in the prototype antiferroelectric Sm- $C_{A}^{*}$ and four-layer antiferroelectric $\frac{1}{2}$ temperature regions [6-9]; hence several closed contour lines with negative EFIB observed in the temperature region between approximately $60{ }^{\circ} \mathrm{C}$ and $75{ }^{\circ} \mathrm{C}$ indicate the emergence of $\frac{1}{2}$ in these temperature regions. The stable antiferroelectric phases have a threshold in the electric-field-induced phase transition to ferroelectric $\mathrm{Sm}-C^{*}$, as clearly observed in the optical transmittance under 

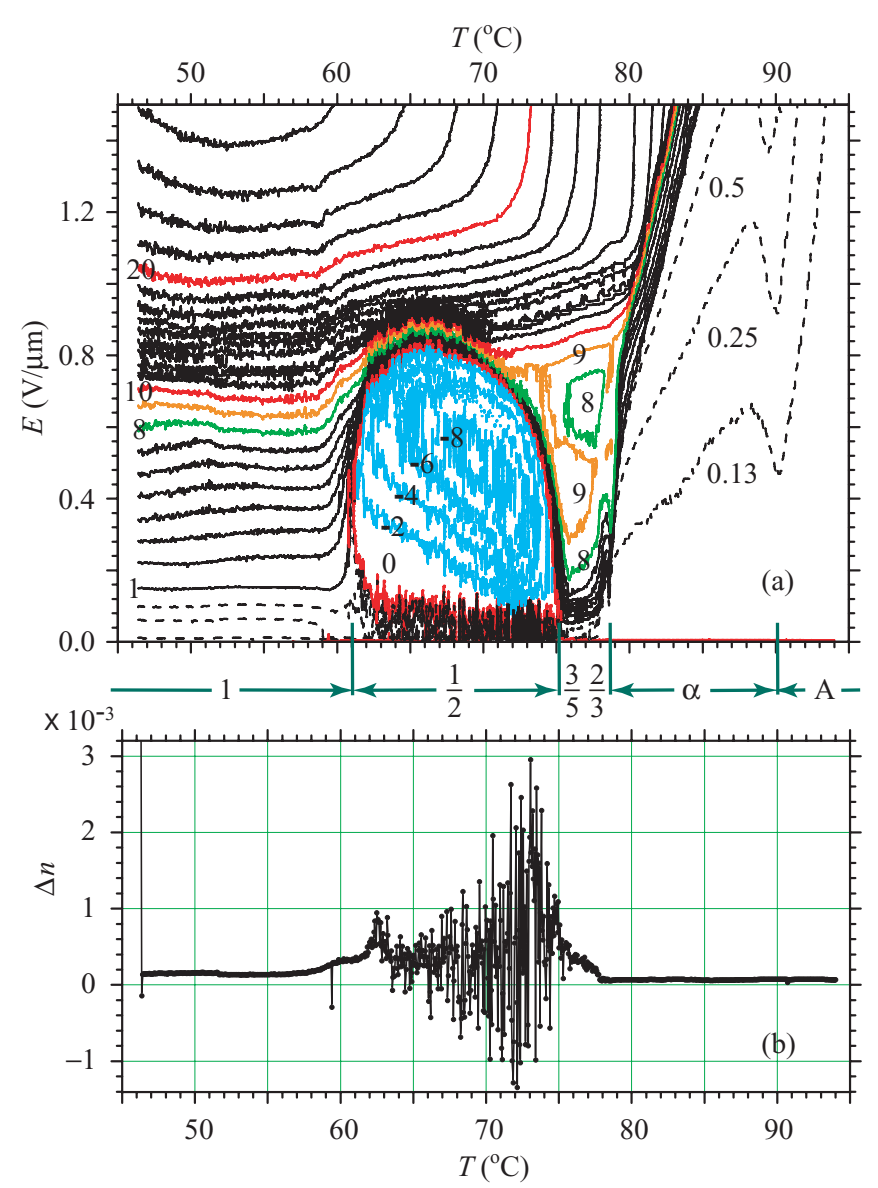

FIG. 3. (Color online) (a) Identification of the six phases given in the lower part of the typical $E-T$ phase diagram for $15.00 \mathrm{wt} \%$ in cooling and (b) EFIB $\Delta n$ vs $T$ at a field of $0.028 \mathrm{~V} / \mu \mathrm{m}$. Here $\alpha$ and $A$ indicate $\mathrm{Sm}-C_{\alpha}^{*}$ and $\mathrm{Sm}-A$, respectively, and the numbers refer to $q_{T}$; note that $\mathrm{Sm}-C^{*}$ has $q_{T}=1$. Contours are drawn by solid lines at an interval of $\Delta n=1 \times 10^{-3}$ and dashed lines show some auxiliary contours in smaller steps of $\Delta n$. Closed contours characteristic of $\frac{1}{2}$ with negative $\Delta n$ values are very noisy and hence drawn at an interval of $\Delta n=-2 \times 10^{-3}$.

crossed polarizers of homogeneously aligned cells [4,39]. As a pretransitional effect, however, a sufficiently small induced polarization forces the alignment of the average tilting direction parallel to the field and causes the apparently continuous buildup of negative EFIB in homeotropically aligned cells [40-42]. The characteristic behavior of EFIB is much more clearly seen in the $\Delta n$ vs $E$ curves given in Fig. 4. To see the signal-to-noise ratio in our EFIB measurements, $\Delta n$ vs $T$ at the minimum applied field of $0.028 \mathrm{~V} / \mu \mathrm{m}$ is shown in Fig. 3(b). The $\frac{1}{2}$ temperature region is very noisy; this may indicate that the macroscopic helix long pitch of $\frac{1}{2}$ is very long. Below four-layer $\frac{1}{2}$, the contours for prototypical $\mathrm{Sm}-C^{*}$ appear in the lowest-temperature region prior to crystallization. Horizontal contours represent the unwinding of the macroscopic long-pitch helix in Sm- $C^{*}$. Rather high fields are required to get unwound $\mathrm{Sm}-C^{*}$; this indicates that the macroscopic helix long pitch must be rather short and is consistent with the fact that the $\Delta n-T$ signal is practically noiseless in Sm- $C^{*}$ as illustrated in Fig. 3(b).

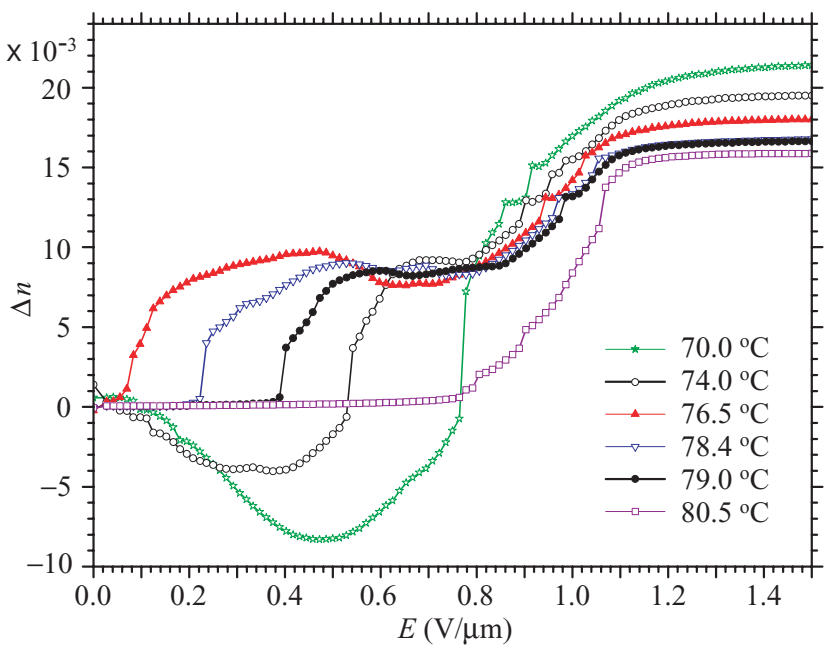

FIG. 4. (Color online) $\Delta n$ vs $E$ curves for selected temperatures: $70.0{ }^{\circ} \mathrm{C}\left(\frac{1}{2}\right), 74.0{ }^{\circ} \mathrm{C}\left(\frac{1}{2}\right), 76.5^{\circ} \mathrm{C}\left(\frac{3}{5}\right), 78.4{ }^{\circ} \mathrm{C}\left(\frac{2}{3}\right), 79.0{ }^{\circ} \mathrm{C}\left(\mathrm{Sm}-C_{\alpha}^{*}\right)$, and $80.5^{\circ} \mathrm{C}\left(\mathrm{Sm}-C_{\alpha}^{*}\right)$. Each selected temperature belongs to the region of the phase at $E=0$ shown in parentheses.

Between $\mathrm{Sm}-C_{\alpha}^{*}$ and $\frac{1}{2}$ we can see two additional temperature regions, which must correspond to six-layer antiferroelectric $\frac{2}{3}$ and the noisy region identified by RXRS [22]. We have assigned the noisy region as five-layer ferrielectric $\frac{3}{5}$ by EFIB [10]. These two were observed in almost all the $E-T$ phase diagrams actually obtained in $11.05,12.45,13.50$, and $15.00 \mathrm{wt}$ \% mixtures, except for the rather fast temperature change of a $0.1{ }^{\circ} \mathrm{C}$ step in $11.05 \mathrm{wt} . \%$ and the heating mode in $15.00 \mathrm{wt}$. \%. Thermal hysteresis is prominent; the two temperature regions are wider in the cooling mode than in the heating mode. In fact, Wang et al. [22] reported results only for the cooling mode. To see more distinctly the characteristic features of $\Delta n$ contour lines in these temperature regions, let us expand the temperature axis of Figs. 2(d1) and 3, the $E-T$ phase diagram of $15.00 \mathrm{wt}$. $\% \mathrm{C} 11$ in cooling, as shown in Fig. 5. Below $\mathrm{Sm}-C_{\alpha}^{*}$, there appears another dent followed by a hump; this indicates the emergence of another phase in a rather narrow temperature range of less than $1{ }^{\circ} \mathrm{C}$. The EFIB contours of this phase are reminiscent of an antiferroelectric phase that has no spontaneous polarization because there exists a conspicuous threshold in a response of EFIB to an applied field. This is much more clearly seen in $\Delta n$ vs $E$ at $78.4{ }^{\circ} \mathrm{C}$ given in Fig. 4. This antiferroelectric phase just below Sm- $C_{\alpha}^{*}$ must be $\frac{2}{3}$, which Wang et al. observed by RXRS, although the temperature range is always less than $1{ }^{\circ} \mathrm{C}$ in all the mixtures investigated and slightly narrower than that Wang et al. reported. One may ask why this antiferroelectric phase $\frac{2}{3}$ does not show any negative EFIB contours, as contrary to the prototypical antiferroelectric phases $\mathrm{Sm}-C_{A}^{*}$ and $\frac{1}{2}$. The reason probably lies in the stability of $\frac{2}{3}$, although the detail has not been studied yet. To show the negative EFIB contours, the antiferroelectric phase needs to be stable enough so that an applied field produces induced spontaneous polarization alone and the average tilting directions orient parallel to the field [40-42]. Actually, however, $\frac{2}{3}$ is not so stable that an applied field deforms its unit cell and produces additional polarization, which compensates for the ordinary induced one. 

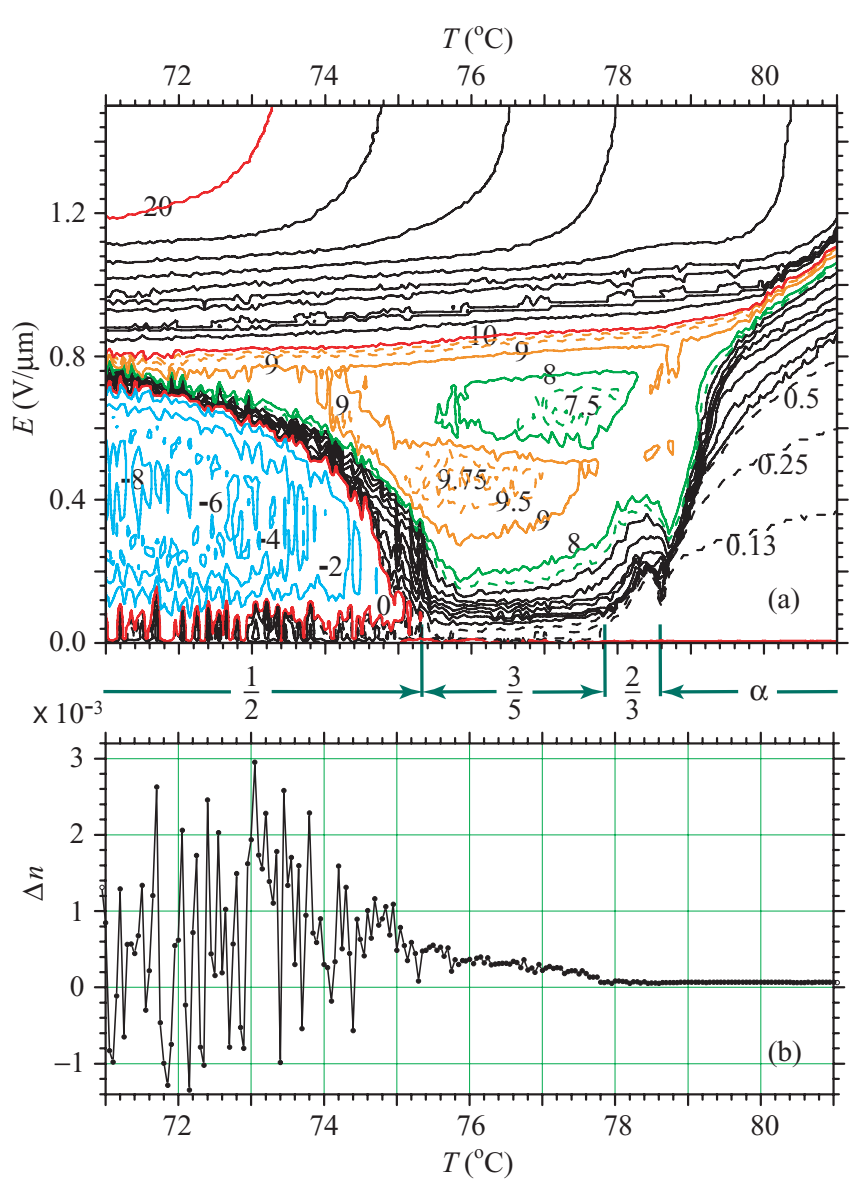

FIG. 5. (Color online) (a) $E-T$ phase diagram of 15.00 wt. \% in cooling with the expanded temperature axis between $71{ }^{\circ} \mathrm{C}$ and $81{ }^{\circ} \mathrm{C}$ and (b) EFIB $\Delta n$ vs $T$ at a field of $0.028 \mathrm{~V} / \mu \mathrm{m}$. Identification of the four phases is given between (a) and (b). Here $\alpha$ indicates Sm- $C_{\alpha}^{*}$ and the numbers refer to $q_{T}$. Contours are drawn by solid lines at an interval of $\Delta n=1 \times 10^{-3}$ and dashed lines show some auxiliary contours in smaller steps of $\Delta n$; closed contours characteristic of $\frac{1}{2}$ with negative $\Delta n$ values are drawn at an interval of $\Delta n=-2 \times 10^{-3}$.

In fact, previously reported unstable $\frac{1}{4}$ also showed a similar behavior $[7,8]$.

On the low-temperature side of the hump, we see the stabilization of another phase in a temperature region of $2{ }^{\circ} \mathrm{C}-3{ }^{\circ} \mathrm{C}$, which corresponds to the noisy region that Wang et al. identified by RXRS [22]. So long as we observe the region with EFIB, it does not look as noisy as the $\frac{1}{2}$ seen in Figs. 3(b) and 5(b) and appears to represent a phase that does have spontaneous polarization. In fact, Chandani et al. identified it as $\frac{3}{5}$ by EFIB as mentioned above [10]. The EFIB increases gradually from zero without showing any conspicuous threshold, as also illustrated in Fig. 4, and the contours are rather parallel to the temperature abscissa axis up to approximately $0.2 \mathrm{~V} / \mu \mathrm{m}$. In this low-field region, an applied field unwinds the macroscopic long-pitch helical structure of the phase. In the high-field region above approximately $1 \mathrm{~V} / \mu \mathrm{m}$, in contrast, the EFIB contours are to be vertical and indicate that the field-induced phase transition occurs to unwound $\mathrm{Sm}-C^{*}$. In the rather wide intermediate-field region in between, the EFIB contours display complex behavior. The EFIB $\Delta n$ does not change much in the region of approximately $0.2-0.8 \mathrm{~V} / \mu \mathrm{m} ; \Delta n=8 \times 10^{-3}$ forms a closed contour and $\Delta n=9 \times 10^{-3}$ is of sigmoid shape, as seen in Fig. 5(a). When we apply higher fields than approximately $0.8 \mathrm{~V} / \mu \mathrm{m}$, the EFIB contours become almost parallel to the temperature axis and $\Delta n$ increases rather steeply until the phase transition occurs to unwound $\mathrm{Sm}-C^{*}$ with the vertical EFIB contours. Figure 4 illustrates the typical behavior of $\Delta n$ vs $E$ at $76.5^{\circ} \mathrm{C}$, which shows the maximum and the minimum and indicates that the transition to unwound $\mathrm{Sm}-\mathrm{C}^{*}$ in this new phase region occurs via at least two field-induced metastable states. Similar behavior suggesting the emergence of metastable states is observed in all the mixtures investigated in both the cooling and heating modes. It is hard to consider that the noisy region consists of the mixture of $\frac{1}{2}$ and $\mathrm{Sm}-C_{\alpha}^{*}$ as reported by Pan et al. [43]. The reason as to why this phase appears noisier in the RXRS experiment will be discussed in Sec. IV A, paragraph 5.

\section{DISCUSSION}

\section{A. Comparison between experimentally obtained $E-T$ phase diagrams with EFIB contours and calculated ones based on the phenomenological Landau model}

Let us begin by summarizing our experimental results of EFIB reported previously [5-11], for the subphases and their emerging sequences, empirically assigned, agree impressively well with those predicted by Dolganov et al. on the basis of the phenomenological Landau model [34-37]. As is well known, EFIB does not tell exactly the superlattice structures of any subphases, although it can rather easily distinguish the antiferroelectric phases from the ferrielectric as well as the ferroelectric ones. To determine the periodicity and the azimuthal distributions of directors in a unit cell of a subphase, we need a model to identify it. Actually, we have so far used the partially molecular Emelyanenko-Osipov model $[3,44]$ plus our own primitive way of understanding the frustration in clinicity. In denominating the subphases (sometimes also called microphases), Dolganov et al. have adopted the number of smectic layers in a unit cell, whereas we have used the $q_{T}$ number. ${ }^{1}$ Since we basically deal with the degeneracy lifting due to any weak LRILIs at the frustration point between anticlinic antiferroelectric and synclinic ferroelectric orderings, $q_{T}$ increases monotonically from 0 in $\mathrm{Sm}-C_{A}^{*}$ to 1 in Sm- $C^{*}$ with rising temperature. Notice that $q_{T}$ is uniquely related to the microscopic distorted-helix short pitch $p_{q T}$ as explained in Sec. I. It is generally considered that subphases

\footnotetext{
${ }^{1}$ In denominating the biaxial subphases Wang et al. used a subscript $d$ (distorted) plus the number of smectic layers in a unit cell or the short pitch of the superlattice structure. As long as we consider $\mathrm{Sm}-C_{d 4}^{*}, \mathrm{Sm}-C_{d 5}^{*}$, and $\mathrm{Sm}-C_{d 6}^{*}$ only, it is not clear whether the number refers to the unit cell size or the short pitch. Dolganov et al. used the same notation and discussed much larger unit cells, e.g., Sm- $C_{d 8}^{*}$, Sm- $C_{d 10}^{*}, \mathrm{Sm}-C_{d 7}^{*}$, etc.; clearly, in denoting these, they considered that this number refers to the unit cell size. When the number indicates the short pitch, these should be written as Sm- $C_{d 8 / 3}^{*}, \mathrm{Sm}-C_{d 10 / 3}^{*}, \mathrm{Sm}-$ $C_{d 7 / 2}^{*}$, etc. Because of the unique relationship between the short pitch and the $q_{T}$ number, we understand that the subscript number Wang et al. used refers to its short pitch.
} 
with smaller unit cells of irreducible $q_{T}$ in lower terms must be observed more easily than the ones with larger unit cells of irreducible $q_{T}$ in higher terms.

In fact, we chose $\frac{1}{2}, \frac{1}{3}, \frac{2}{3}, \frac{1}{4}, \frac{3}{4}, \frac{1}{5}, \frac{2}{5}, \frac{3}{5}$, and $\frac{4}{5}$ as possible $q_{T}$ representing smaller unit cells, which may be described as $(F A),(F A A),(F F A),(F A A A),(F F F A)$, $(F A A A A),(F A F A A),(F F A F A)$, and $(F F F F A)$. Here $F$ and $A$ refer to the quasisynclinic ferroelectric and the quasianticlinic antiferroelectric orderings, respectively. The actual unit cell of $q_{T}=m / n$ with even $m$ or $n$ should contain $2 n$ smectic layers and the subphase is antiferroelectric; otherwise it could not maintain periodicity. When both $m$ and $n$ are odd, the denominator represents the number of smectic layers contained in the unit cell and the subphase is ferrielectric. The first three $\frac{1}{2}, \frac{1}{3}$, and $\frac{2}{3}$ were confirmed to exist and their structures were clarified by RXRS and other sophisticated experimental techniques [16-22]; these are the four-layer antiferroelectric, the three-layer ferrielectric, and the six-layer antiferroelectric subphases, respectively. The next one $\frac{1}{4}$ is the eight-layer antiferroelectric subphase with a microscopic distorted-helix short pitch of $8 / 3$. Based on our experimental observations, we are confident that such a subphase was observed in Figs. 6-9 of Ref. [7] and Fig. 5 of Ref. [8]; its emergence was confirmed in different materials by several experimental methods. Regarding $\frac{1}{5}$, we referred to the existence in Fig. 2 of Ref. [5], Fig. 2 curve (d) of Ref. [12], and Fig. 6 of Ref. [8]; the evidence in these cases is rather indirect though. We also referred to the emergence of $\frac{2}{5}$, the ten-layer antiferroelectric subphase, in Fig. 3 of Ref. [8], although it is not easy to differentiate $\frac{2}{5}$ from the tail of $\frac{1}{2}$ unambiguously. The pattern of $\frac{3}{5}$ in Fig. 4 of Ref. [8] is so characteristic that we can believe its existence as the five-layer ferrielectric subphase. Experimentally, seven subphases with $q_{T}=\frac{1}{5}, \frac{1}{4}, \frac{1}{3}, \frac{2}{5}, \frac{1}{2}, \frac{3}{5}$, and $\frac{2}{3}$ are considered to exist.

Dolganov et al. [35] showed that, except for $\frac{1}{5}$, the remaining six of these emerge in sequences similar to those experimentally observed. They used the phenomenological Landau theory of the phase transitions with a two-component order parameter $\boldsymbol{\xi}_{i}$; but they did not take into account the Lifshitz term related to chirality, $f\left[\boldsymbol{\xi}_{i} \times \boldsymbol{\xi}_{i+1}\right]_{z}$, which may not in any case change the number of phases and their temperature sequences. The Landau expansion of the free energy is written as $F=F_{1}+F_{2}+F_{3}+F_{4}$, where

$$
\begin{gathered}
F_{1}=\sum_{i}\left[\frac{1}{2} \alpha\left(T-T^{*}\right) \xi_{i}^{2}+\frac{1}{4} b_{0} \xi_{i}^{4}\right] \\
F_{2}=\frac{1}{2} a_{1} \sum_{i} \xi_{i} \cdot \xi_{i+1}+\frac{1}{8} a_{2} \sum_{i} \xi_{i} \cdot \xi_{i+2} \\
+b \sum_{i}\left[\xi_{i} \times \xi_{i+1}\right]^{2} \\
F_{3}=b_{1} \sum_{i} \xi_{i}^{2}\left(\xi_{i-1} \cdot \xi_{i}+\xi_{i} \cdot \xi_{i+1}\right)+a_{3} \sum_{i} \xi_{i} \cdot \xi_{i+3},
\end{gathered}
$$

and

$$
F_{4}=\sum_{i} P_{0} E \xi_{i} \sin \varphi_{i} .
$$

They analyzed Sm- $C_{A}^{*}-\mathrm{Sm}-A$ and $\mathrm{Sm}-C^{*}-\mathrm{Sm}-A$ phase transitions at zero field by neglecting $F_{3}$ and using $F=F_{1}+F_{2}$ and showed that a pair of main phases and subphases with the same value of $\left|q_{T}-\frac{1}{2}\right|$, such as $\mathrm{Sm}-C_{A}^{*}$ and $\mathrm{Sm}-C^{*}$ (i.e., $q_{T}=0$ and 1), $\frac{1}{3}$ and $\frac{2}{3}, \frac{2}{5}$ and $\frac{3}{5}, \frac{3}{7}$ and $\frac{4}{7}$, etc., have the same free energy and also clarified their characteristic superlattice structures. Then they took into account $F_{3}$ given in Eq. (3) and obtained the two typical (so they claimed ${ }^{2}$ ) phase emerging sequences Sm- $C^{*}-\frac{1}{2}-\frac{3}{5}-\frac{2}{3}-\mathrm{Sm}-C_{\alpha}^{*}-\mathrm{Sm}-A$ and $\mathrm{Sm}-C_{A}^{*}-\frac{1}{4}-\frac{1}{3}-\frac{2}{5}-\frac{1}{2}-\mathrm{Sm}-C^{*}-\mathrm{Sm}-A$, together with their characteristic superlattice structures.

In this way, the general tendency of the emerging sequences of subphases as well as their superlattice structures at zero field, which have been identified experimentally [5-12] so far, was reproduced by minimizing the free energy $F=F_{1}+F_{2}+F_{3}$ given in Eqs. (1)-(3). Note that similar free-energy terms had been used by several researchers without systematically clarifying the subphase superlattice structures other than those of $\frac{1}{3}$ and $\frac{1}{2}$ as well as the staircase character of the emerging sequences. Herein lies the difference: The minimization of the Landau free energy was performed by Dolganov et al. over a set of tilt angle $\theta_{i}$ and azimuthal angle $\varphi_{i}$ in subphase superlattice structures with larger than three- and four-layer unit cells, whereas it had been done with respect to the azimuthal angle $\varphi_{i}$ alone by other researchers [45-48]. In the subphase superlattice structures Dolganov et al. thus obtained, not only the azimuthal angle $\varphi_{i}$ but also the tilt angle $\theta_{i}$ changes from layer to layer within the unit cell. In fact, these superlattice structures with the spatial variation of both $\theta_{i}$ and $\varphi_{i}$ are very characteristic and should be clearly confirmed experimentally in a subphase such as $q_{T}=\frac{1}{4}$ where the tilt angle $\theta_{i}$ is rather large. It is also worthwhile trying to reproduce a number of emerging phase sequences experimentally observed [5-12] by properly choosing coefficients in the Landau theory. These problems are left for future study.

Now let us proceed to a comparison between the experimentally obtained $E-T$ phase diagram with EFIB contours with the calculated one from the phenomenological Landau model. The subphase emerging sequence at zero field identified experimentally in Sec. III is well reproduced by the numerically calculated one shown in Fig. 3 of Ref. [34]. The difference lies in the temperature ranges of $\frac{3}{5}$ and $\frac{2}{3}$; experimentally $\frac{3}{5}$ is wider than $\frac{2}{3}$, as seen in Fig. 5, whereas the situation is opposite in the numerically calculated $E-T$ phase diagram. The stability range of a subphase may critically depend on a particular material studied. In the mixture system of 1-trifluoromethylundecyl4-(4'-dodecyloxybiphenyl-4-yl-carbonloxy)-3-fluorobenzoate (12BIMF10) and 4-(1-methylheptyloxycarbonyl)-phenyl4 -octylbiphenyl-4-carboxylate (MHPBC), for example, the phase emerging sequence $\mathrm{Sm}-C_{A}^{*}-\frac{1}{4}-\mathrm{Sm}-C_{\alpha}^{*}$ is observed in pure 12BIMF10; with increasing MHPBC concentration, first $\frac{1}{3}$ and then $\frac{1}{2}$ appear in the emerging sequence of Sm- $C_{A}^{*}-\frac{1}{4}-\frac{1}{3}-\frac{1}{2}-\mathrm{Sm}-C_{\alpha}^{*}$ [7]. Since there are many adjustable parameters in the phenomenological Landau model, it is not impertinent to consider that the observed stability ranges can better be reproduced by properly choosing the parameter

\footnotetext{
${ }^{2}$ The emergence of $\frac{1}{2}$ on the high-temperature side of Sm- $C^{*}$ is not typical. We insist that this emerging sequence is quite unusual and extraordinary, as discussed in Sec. IV B.
} 
values. Regarding the noisy region Wang et al. identified [22], we would like to point out that $\frac{3}{5}$ is ferrielectric and that any ferrielectric phase may be noisier. Even in the well established ferrielectric subphase $\frac{1}{3}$, the disordered structure and lowfrequency fluctuations were observed by laser beam diffraction and photon correlation spectroscopy [49]. This intrinsic instability has been ascribed to a tendency of neutralizing the spontaneous polarization spatially and thus reducing the Coulomb interaction energy; solitonlike mismatches are easily produced here and there by interchanging the antiferroelectric and ferroelectric orderings, thus causing thermal fluctuations in the helicoidal structures [49]. Levelut and Pansu also wrote that the RXRS experiments have revealed the existence of defects in $\frac{1}{3}$ since the resonant satellite peaks show a complex structure with several side bands [15].

As predicted by Dolganov et al., the temperature range of $q_{T}=\frac{3}{5}$ at zero field apparently widens into both lowand high-temperature sides with increasing applied field up to $\sim 0.8 / \mathrm{V} / \mu \mathrm{m}$. Within this widened area, the contours of birefringence are rather parallel to the temperature abscissa axis. In the low field up to $\sim 0.1 \mathrm{~V} / \mu \mathrm{m}$, the widening is not conspicuous and the contours are dense, where the macroscopic long-pitch helical structure is considered to be unwinding. Then the widening becomes conspicuous particularly on the low-temperature side; the contours are coarse and draw closed loops or sigmoid-type curves. This means that the applied field first stabilizes unwound $\frac{3}{5}$ and then causes the transition to another field-induced subphase. The changes in the structures are more clearly seen in Fig. 4, where EFIB $\Delta n$ at a particular temperature is plotted as a function of an applied field $E$. At $76.5{ }^{\circ} \mathrm{C}$ in the middle of the subphase $q_{T}=\frac{3}{5}, \Delta n$ shows the maximum at $E \approx 0.47 \mathrm{~V} / \mu \mathrm{m}$ and the minimum at $E \approx 0.66 \mathrm{~V} / \mu \mathrm{m}$; the maximum represents unwound $\frac{3}{5}$ and the minimum must correspond to a field-induced subphase. Increasing the applied field produces nearly the same effect as increasing temperature in the sense that both favor the ferroelectric state. Since the applied field selectively determines the director tilting sense, we should use the number $q_{E}$, such that

$$
q_{E}=\frac{|[R]-[L]|}{[R]+[L]},
$$

instead of the $q_{T}$ number. Here $[R]$ and $[L]$ refer to the numbers of smectic layers with directors tilted to the right and to the left, respectively, in the unit cell of a field-induced subphase. In the case of five-layer unit cells, two field-induced subphases are conceivable as shown in Fig. 6. The field-induced transition from $q_{E}=\frac{1}{5}\left(q_{T}=\frac{3}{5}\right)$ to $q_{E}=\frac{3}{5}$ may easily occur, for only one-layer flipping in the unit cell causes this transition. It is not impertinent to assign the minimum at $76.5{ }^{\circ} \mathrm{C}$ in Fig. 4 to $q_{E}=\frac{3}{5}$. In the case of the usual ordinary phase sequence, the similar maximum and minimum were observed as shown in Fig. 4 of Ref. [8], although a detailed study has not been done yet.

Such a staircase character of the field-induced transitions can be seen in all the subphase regions at zero field, $q_{T}=\frac{1}{2}$, $\frac{3}{5}, \frac{2}{3}$, and $\mathrm{Sm}-C_{\alpha}^{*}$, in Fig. 5. In the high-temperature part of $q_{T}=\frac{1}{2}$, e.g., at $74.0{ }^{\circ} \mathrm{C}$, the field-induced transition from $q_{E}=0\left(q_{T}=\frac{1}{2}\right)$ to $q_{E}=1\left(q_{T}=1\right.$, i.e., Sm- $\left.C^{*}\right)$ appears to

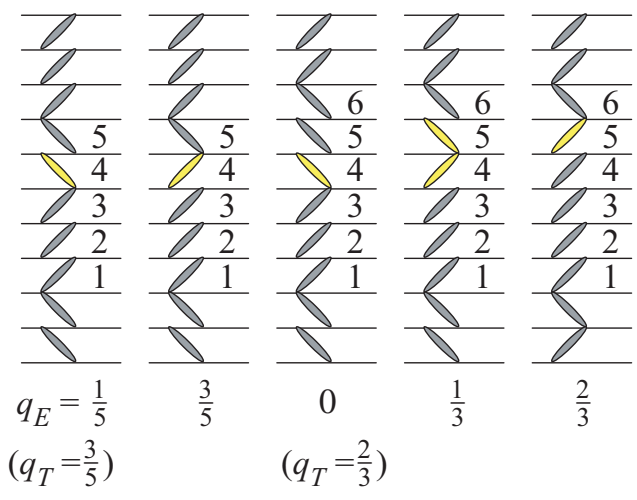

FIG. 6. (Color online) Schematically illustrated planar projections of unit-cell structures for field- and temperature-induced subphases. The top views of the field-induced subphases have not been calculated yet, although the temperature-induced ones of $q_{T}=\frac{3}{5}$ and $\frac{2}{3}$ were given in Ref. [50].

occur at least via $q_{E}=\frac{1}{5}$ and/or $\frac{3}{5}$. In the usual ordinary phase sequence, RXRS clearly reveals the field-induced sequential transitions $q_{E}=0\left(q_{T}=\frac{1}{2}\right)$ to $q_{E}=\frac{1}{3}\left(q_{T}=\frac{1}{3}\right)$ to $q_{E}=1$ $\left(q_{T}=1\right.$, i.e., Sm- $\left.C^{*}\right)$ [21,51]; the $E-T$ phase diagram with EFIB contours given in Fig. 2(i) of Ref. [8] also suggested several (possibly metastable) field-induced subphases between $q_{E}=0\left(q_{T}=\frac{1}{2}\right)$ and $q_{E}=1\left(q_{T}=1\right.$, i.e., Sm- $\left.C^{*}\right)$. In the temperature region of $q_{T}=\frac{2}{3}$, the situation is much more complex: The field-induced transition from $q_{E}=0\left(q_{T}=\frac{2}{3}\right)$ to $q_{E}=1\left(q_{T}=1\right.$, i.e., $\left.\mathrm{Sm}-C^{*}\right)$ appears to occur at least via some of $q_{E}=\frac{1}{5}, \frac{1}{3}, \frac{3}{5}$, and $\frac{2}{3}$ as given in Fig. 6. Even in the low-temperature part of $\mathrm{Sm}-C_{\alpha}^{*}$, e.g., at $79.0^{\circ} \mathrm{C}$, the field-induced transition to $\mathrm{Sm}-C^{*}$ does not occur in a single step, for the boundary between $q_{T}=\frac{2}{3}$ and $\mathrm{Sm}-C_{\alpha}^{*}$ is not vertical, contrary to the calculated $E-T$ phase diagram given in Fig. 3 of Ref. [34]. In the usual ordinary phase sequence, a more prominent nonvertical boundary between $q_{T}=\frac{3}{5}$ and $\mathrm{Sm}-C_{\alpha}^{*}$ was observed in Fig. 4 of Ref. [8]; furthermore, some staircase characters were observed in the field-induced unwinding process of Sm- $C_{\alpha}^{*}$ given in Figs. 2(a), 2(b), 2(e), and 2(f) of Ref. [8] and preliminary theoretical investigations were performed [52-55]. In this way many intriguing preliminary data and theoretical analyses concerning the field-induced sequential transitions are waiting for full detailed systematic investigations.

\section{B. Extent to which the quasimolecular Emelyanenko-Osipov model can explain the unusual extraordinary phase sequence}

In the unusual extraordinary phase sequence, we can consider that $\mathrm{Sm}-C^{*}$ and $\mathrm{Sm}-A$ are frustrating; then $F_{i}$ corresponding to Eq. (49) of Ref. [3], which represents SRILIs, can be written as

$$
\begin{aligned}
F_{i}= & \alpha \frac{T-T_{\mathrm{c}}}{T_{\mathrm{c}}} \sin ^{2} \theta_{i}+B \sin ^{4} \theta_{i} \\
& -a \sin ^{2} \theta_{i} \cos ^{2} \theta_{i}\left(\cos \varphi_{i-1, i}+\cos \varphi_{i, i+1}\right) \\
& -b \sin ^{4} \theta_{i}\left(\cos ^{2} \varphi_{i-1, i}+\cos ^{2} \varphi_{i, i+1}\right),
\end{aligned}
$$

where $T_{\mathrm{c}}$ is approximately equal to the $\mathrm{Sm}-A-\mathrm{Sm}-C^{*}$ phase transition temperature, $a>0$, and $b>0$. In contrast, we try 
to use the effective LRILIs

$$
\begin{aligned}
\Delta F_{i}= & \frac{1}{2 \chi}\left\{\mathbf{P}_{i}^{2}+g\left(\mathbf{P}_{i-1} \cdot \mathbf{P}_{i}+\mathbf{P}_{i} \cdot \mathbf{P}_{i+1}\right)\right\} \\
& +c_{\mathrm{p}}\left(\mathbf{P}_{i} \cdot \boldsymbol{\xi}_{i}\right)+c_{\mathrm{f}}^{(1)} \cos \theta_{i}\left(\mathbf{P}_{i} \cdot \Delta \mathbf{n}_{i \pm 1}\right) \\
& +c_{\mathrm{f}}^{(2)} \cos \theta_{i}\left(\mathbf{P}_{i} \cdot \mathbf{n}_{i}\right)\left(\Delta \mathbf{n}_{i \pm 1} \cdot \mathbf{n}_{i}\right),
\end{aligned}
$$

as pointed out in Sec. I. Actually, we assume that the total polarization of a smectic layer may always be parallel to the layer plane and neglect the second flexoelectric term, setting $c_{\mathrm{f}}^{(1)}=c_{\mathrm{f}}$, in the following. By minimizing the total free energy

$$
F=\sum_{i=1}^{N}\left(F_{i}+\Delta F_{i}\right),
$$

first with respect to the polarization $\mathbf{P}_{i}$ and then with respect to the tilt angle $\theta_{i}$ and the azimuthal angle $\varphi_{i}$, we can determine their temperature dependences. Even the tilt angle may not be spatially uniform and we may need to consider its dependence on $i$. In the biaxial subphases experimentally studied so far [22], however, $\theta_{i}$ does not appear to change much from layer to layer; moreover, in optically uniaxial $\mathrm{Sm}-C_{\alpha}^{*}$, the tilt angle monotonically decreases to zero as the temperature increases toward $T_{\mathrm{c}}$. Therefore, we approximately use the spatially uniform tilt angle $\theta$ as the effective temperature in the following. Thus we can avoid the complexity of determining the temperature dependence of $\theta$ that can properly reproduce the dependence actually observed [56,57].

It was shown in the Appendix of Ref. [8] that in the case of Sm- $C_{\alpha}^{*}$ where $\varphi_{i-1, i}=\varphi_{i, i+1}=\varphi=$ const, Eq. (7) is written as

$$
\Delta F_{i}=-\frac{\chi \sin ^{2} \theta \cos ^{2} \theta\left(c_{\mathrm{p}}-2 c_{\mathrm{f}} \sin \varphi\right)^{2}}{2(1+2 g \cos \varphi)} .
$$

The modified free energy of $\mathrm{Sm}-C_{\alpha}^{*}$ per single layer can be represented by

$$
\begin{aligned}
& \frac{F_{\alpha}-F_{0}(\theta)}{2 a \sin ^{2} \theta \cos ^{2} \theta} \\
& =-\cos \varphi-(b / a) \tan ^{2} \theta \cos ^{2} \varphi \\
& \quad-\left(\chi c_{\mathrm{p}} c_{\mathrm{f}} / a\right) \frac{\left(c_{\mathrm{p}} / c_{\mathrm{f}}\right) / 4-\sin \varphi+\left(c_{\mathrm{f}} / c_{\mathrm{p}}\right) \sin ^{2} \varphi}{1+2 g \cos \varphi} .
\end{aligned}
$$

By reference to Eqs. (61)-(63) of Ref. [3], the modified free energy per single layer of a biaxial subphase with a unit cell of $t$ layers is represented by

$$
\begin{aligned}
& \frac{F_{t}-F_{0}(\theta)}{2 a \sin ^{2} \theta \cos ^{2} \theta} \\
& =-\frac{1}{t} \sum_{i=0}^{t-1} \cos \varphi_{i, i+1}-\frac{(b / a)}{t} \tan ^{2} \theta \sum_{i=0}^{t-1} \cos ^{2} \varphi_{i, i+1} \\
& \quad-\frac{\left(\chi c_{\mathrm{p}} c_{\mathrm{f}} / a\right)}{4}\left\{\left(c_{\mathrm{p}} / c_{\mathrm{f}}\right) f_{0}+\left(c_{\mathrm{f}} / c_{\mathrm{p}}\right) f_{0}^{(2)}\right\} \\
& \quad-\frac{\left(\chi c_{\mathrm{p}} c_{\mathrm{f}} / a\right)}{2 t} \sum_{i=0}^{t-2} \sum_{j=i+1}^{t-1}\left\{\left(c_{\mathrm{p}} / c_{\mathrm{f}}\right) f_{j-i}+\left(c_{\mathrm{f}} / c_{\mathrm{p}}\right) f_{j-i}^{(2)}\right\} \cos \varphi_{i, j} \\
& +\frac{\left(\chi c_{\mathrm{p}} c_{\mathrm{f}} / a\right)}{t} \sum_{i=0}^{t-2} \sum_{j=i+1}^{t-1} f_{j-i}^{(1)} \sin \varphi_{i, j} .
\end{aligned}
$$

Biaxial subphases are nonplanar, but the actual structure does not deviate strongly from the corresponding planar prototype. Given the clinicity of a $t$-layer unit cell by assigning 0 or $\pi$ for $\alpha_{i}^{0} \equiv \varphi_{i, i+1}^{0}$, Eqs. (64)-(69) of Ref. [3] ensure that we can uniquely determine the small deviation angles $\Delta \alpha_{i} \equiv \Delta \varphi_{i, i+1}$ that minimize the modified free energy of Eq. (11). After checking all the possible sequences of $\alpha_{i}^{0}$, we obtain the optimal structure of the biaxial subphase with the $t$-layer unit cell. Finally, the free energies of phases with different-size unit cells as well as of $\mathrm{Sm}-C_{\alpha}^{*}$ and $\mathrm{Sm}-C^{*}$ are compared with one another to select the phase that corresponds to the global minimum at a given tilt angle $\theta$ for a particular choice of the model parameters.

In addition to the tilt angle $\theta$ that plays a role in the temperature, there exist four dimensionless parameters $b / a$, the ratio of $b$ to $a$ in Eq. (6); $c_{\mathrm{f}} / c_{\mathrm{p}}$, the ratio between flexoelectric and piezoelectric coefficients; $\chi c_{\mathrm{p}} c_{\mathrm{f}} / a$, the strength of LRILIs as compared to that of SRILIs; and $g$, the molecular positional correlation in adjacent layers. If we use the modified tilt angle

$$
\tilde{\theta}=(b / a) \tan ^{2} \theta
$$

instead of $\theta$ itself, we can further reduce the number of dimensionless parameters to three and obtain the $g-\tilde{\theta}$ phase diagrams given in Fig. 7, which correspond to the prototypical
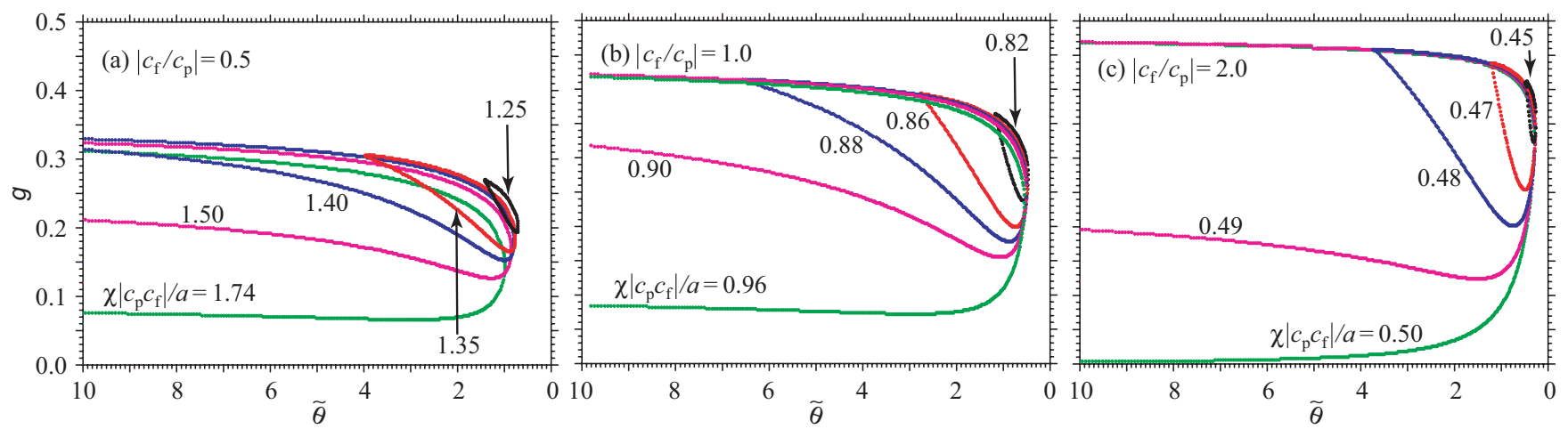

FIG. 7. (Color online) $g-\tilde{\theta}$ phase diagrams in three $\left|c_{\mathrm{f}} / c_{\mathrm{p}}\right|$ : (a) 0.5 , (b) 1.0 , and (c) 2.0. The sign of $c_{\mathrm{f}} c_{\mathrm{p}}$ determines the handedness of the microscopic short-pitch helix; plus and minus refer to left- and right-handed, respectively. For $\chi\left|c_{\mathrm{p}} c_{\mathrm{f}}\right| / a$ larger than a critical value that depends on $\left|c_{\mathrm{f}} / c_{\mathrm{p}}\right|, \frac{1}{2}$ is stabilized; it starts to emerge within an area surrounded by a droplet-shaped curve and then grows into a larger $\tilde{\theta}$ area as $\chi\left|c_{\mathrm{p}} c_{\mathrm{f}}\right| / a$ increases. 


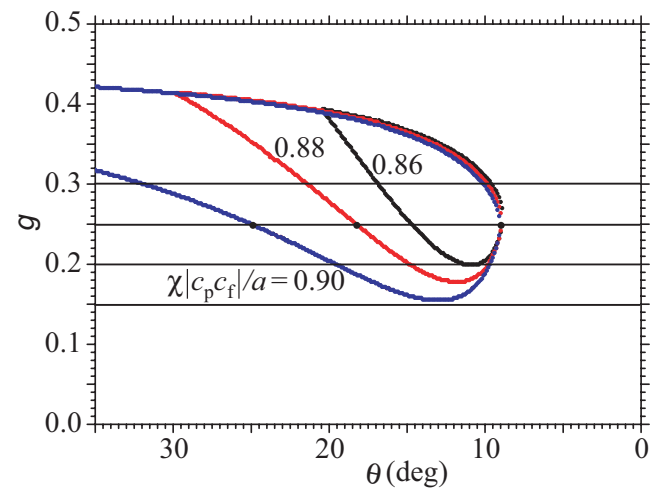

FIG. 8. (Color online) $g-\theta$ phase diagrams. The parameters used are $a / b=0.05,\left|c_{\mathrm{f}} / c_{\mathrm{p}}\right|=1.0$, and $\chi\left|c_{\mathrm{p}} c_{\mathrm{f}}\right| / a=0.86,0.88$, and 0.90 .

$g-\tilde{T}$ phase diagrams given in Fig. 6 of Ref. [3] and Fig. 11 of Ref. [11] in the usual ordinary phase sequence. The biaxial four-layer phase $\frac{1}{2}$ alone is stabilized and any other biaxial subphases do not emerge. Notice that the unusual extraordinary phase sequence $\mathrm{Sm}-C^{*}-\frac{1}{2}-\mathrm{Sm}-C_{\alpha}^{*}$ with rising temperature (with decreasing modified tilt angle $\tilde{\theta}$ ) is observed only when each of $c_{\mathrm{f}} / c_{\mathrm{p}}, \chi c_{\mathrm{p}} c_{\mathrm{f}} / a$, and $g$ has an appropriate value belonging to a rather narrow range. This restriction on parameter values is anticipated because otherwise the extraordinary phase sequence would be observed much more frequently. In fact, the ordinary phase sequence was discovered long ago and is observed in numerous compounds and mixtures, whereas the extraordinary one has been observed only recently in a limited number of materials.

Now let us consider the temperature dependence of the $\mathrm{Sm}-C_{\alpha}^{*}$ microscopic helix short-pitch more intuitively by using the tilt angle $\theta$ itself instead of the modified one $\tilde{\theta}$. Here we assume $\left|c_{\mathrm{f}} / c_{\mathrm{p}}\right|=1.0$ and $a / b=0.05$ as their realistic values; we judge that the piezoelectric and flexoelectric effects are of the same order of magnitude [3] and that $\mathrm{Sm}-C_{\alpha}^{*}$ is stabilized when the tilt angle becomes smaller than approximately $10^{\circ}$ [58,59]. Thus we obtain Fig. 8, which is the same as Fig. 7(b) with $a / b=0.05$. When a line parallel to the abscissa axis, e.g., $g=0.20,0.25$, or 0.30 , crosses the droplet-shaped curve, the extraordinary phase sequence $\mathrm{Sm}-C^{*}-\frac{1}{2}-\mathrm{Sm}-C_{\alpha}^{*}$ is observed as the tilt angle $\theta$ decreases (as the temperature rises); otherwise a continuous change occurs from $\mathrm{Sm}-C^{*}$ to $\mathrm{Sm}-C_{\alpha}^{*}$. Table I lists the tilt angle $\theta$ (deg), at which the phase transitions Sm$C^{*}-\frac{1}{2}$ and $\frac{1}{2}-\mathrm{Sm}-C_{\alpha}^{*}$ occur for $g=0.20,0.25$, and 0.30 and $\chi\left|c_{\mathrm{p}} c_{\mathrm{f}}\right| / a=0.86,0.88$, and 0.90 .

The twisting angle per layer of $\mathrm{Sm}-C_{\alpha}^{*}$ is calculated as the $\varphi$ value that minimizes the modified free energy given in Eq. (10). Table II summarizes the twisting angle per layer for parameter values used to obtain Table I and Fig. 8. Notice that the twisting angle $\varphi$ listed in Table II does not depend on $a / b$, although the tilt angle $\theta$ listed in Table I naturally depends on it. When a line with a particular value of $g$ crosses a droplet-shaped boundary curve at the smallest modified tilt angle $\tilde{\theta}$, the twisting angle $\varphi$ stays constant $90^{\circ}$ with decreasing $\theta$. Below this $g$ value the twisting angle $\varphi$ at the phase transition between $\frac{1}{2}$ and $\mathrm{Sm}-C_{\alpha}^{*}$ is smaller than $90^{\circ}$ and increases with decreasing $\theta$, whereas above this $g$ value it is larger than $90^{\circ}$ and decreases with decreasing $\theta$. In fact, in Table II, the twisting angle $\varphi$ stays
TABLE I. Tilt angle $\theta(\mathrm{deg})$ at which the phase transitions Sm$C^{*}-\frac{1}{2}$ and $\frac{1}{2}-\mathrm{Sm}-C_{\alpha}^{*}$ occur for $a / b=0.05,\left|c_{\mathrm{f}} / c_{\mathrm{p}}\right|=1.0, g=0.20$, 0.25 , and 0.30 , and $\chi\left|c_{\mathrm{p}} c_{\mathrm{f}}\right| / a=0.86,0.88$, and 0.90 .

\begin{tabular}{cccc}
\hline \hline$g$ & & $\begin{array}{c}\text { Sm- } C^{*}-\frac{1}{2} \\
\theta\end{array}$ & $\begin{array}{c}\frac{1}{2}-\mathrm{Sm}-C_{\alpha}^{*} \\
\theta\end{array}$ \\
\hline 0.20 & $\chi\left|c_{\mathrm{p}} c_{\mathrm{f}}\right| / a$ & $11.4^{\circ}$ & $10.4^{\circ}$ \\
0.20 & 0.86 & $14.9^{\circ}$ & $9.8^{\circ}$ \\
0.20 & 0.88 & $19.6^{\circ}$ & $9.7^{\circ}$ \\
0.25 & 0.90 & $14.7^{\circ}$ & $8.9^{\circ}$ \\
0.25 & 0.86 & $18.3^{\circ}$ & $9.0^{\circ}$ \\
0.25 & 0.88 & $24.9^{\circ}$ & $9.1^{\circ}$ \\
0.30 & 0.90 & $16.8^{\circ}$ & $9.6^{\circ}$ \\
0.30 & 0.86 & $21.3^{\circ}$ & $9.8^{\circ}$ \\
0.30 & 0.88 & $31.8^{\circ}$ & $10.1^{\circ}$ \\
\hline \hline
\end{tabular}

almost constant at around $90^{\circ}$ for $g=0.25$, which crosses each of the droplet-shaped boundary curves with $\chi\left|c_{\mathrm{p}} c_{\mathrm{f}}\right| / a=0.86$, 0.88 , and 0.90 near the smallest tilt angle as shown in Fig. 8, whereas it increases for $g=0.2$ with rising temperature (i.e., with decreasing $\theta$ ) and decreases for $g=0.3$, but does not become smaller than $90^{\circ}$. For other parameter values of $\left|c_{\mathrm{f}} / c_{\mathrm{p}}\right|$, it hardly reproduces the results of Wang et al. According to their results summarized in Fig. 2(b) of Ref. [22], the short pitch at the phase transition temperature between $\mathrm{Sm}-C_{\alpha}^{*}$ and Sm- $A$, i.e., at $\theta=0$, is about $p_{\alpha}=6.7$ layers $\left(\varphi=53.7^{\circ}\right)$ and gradually becomes shorter to be 5.5 layers $\left(\varphi=65.5^{\circ}\right)$ at the Sm- $C_{\alpha}^{*}-\frac{2}{3}$ phase transition temperature. We can extrapolate it down to the $\frac{1}{2}$ phase transition temperature and obtain 5.3 layers $\left(\varphi=67.9^{\circ}\right)$.

It is easily foreseeable that the modified free energy of Sm- $C_{\alpha}^{*}$ given by Eq. (10) cannot reproduce the experimentally observed temperature dependence of the Sm- $C_{\alpha}^{*}$ microscopic helix short pitch. Equation (10) consists of two parts: the polarization-independent part responsible for the SRILIs and the polarization-dependent part responsible for the effective LRILIs, both of which are drawn in Fig. 9 for typical parameter values. Since the minimum of the polarization-dependent LRILI part occurs at around $\varphi \simeq 90^{\circ}$, the $\mathrm{Sm}-C_{\alpha}^{*}$ twisting

TABLE II. Twisting angle per layer $\varphi(\mathrm{deg})$, or short pitch $p_{\alpha}$ (smectic layers per turn), of Sm- $C_{\alpha}^{*}$ at the temperatures (tilt angles) where the phase transitions $\frac{1}{2}-\mathrm{Sm}-C_{\alpha}^{*}$ and Sm- $C_{\alpha}^{*}-\mathrm{Sm}-A$ occur for $\left|c_{\mathrm{f}} / c_{\mathrm{p}}\right|=1.0, g=0.20,0.25$, and 0.30 , and $\chi\left|c_{\mathrm{p}} c_{\mathrm{f}}\right| / a=0.86,0.88$, and 0.90 .

\begin{tabular}{lccc}
\hline \hline$g$ & $\chi c_{\mathrm{p}} c_{\mathrm{f}} / a$ & $\begin{array}{c}\frac{1}{2}-\mathrm{Sm}-C_{\alpha}^{*} \\
\varphi\left(p_{\alpha}\right)\end{array}$ & $\begin{array}{c}\text { Sm- } C_{\alpha}^{*}-\mathrm{Sm}-A \\
\varphi\left(p_{\alpha}\right)\end{array}$ \\
\hline 0.20 & 0.86 & $42.0^{\circ}$ (8.6 layers) & $82.9^{\circ}$ (4.3 layers) \\
0.20 & 0.88 & $63.4^{\circ}$ (5.7 layers) & $83.7^{\circ}$ (4.3 layers) \\
0.20 & 0.90 & $71.2^{\circ}$ (5.1 layers) & $84^{\circ}$ (4.3 layers) \\
0.25 & 0.86 & $86.7^{\circ}$ (4.2 layers) & $88.8^{\circ}$ (4.1 layers) \\
0.25 & 0.88 & $89.1^{\circ}$ (4.0 layers) & $89.7^{\circ}$ (4.0 layers) \\
0.25 & 0.90 & $91.0^{\circ}$ (4.0 layers) & $90.4^{\circ}$ (4.0 layers) \\
0.30 & 0.86 & $107.8^{\circ}$ (3.3 layers) & $96.9^{\circ}$ (3.7 layers) \\
0.30 & 0.88 & $109.2^{\circ}$ (3.3 layers) & $97.8^{\circ}$ (3.7 layers) \\
0.30 & 0.90 & $110.8^{\circ}$ (3.2 layers) & $98.6^{\circ}$ (3.7 layers) \\
\hline \hline
\end{tabular}



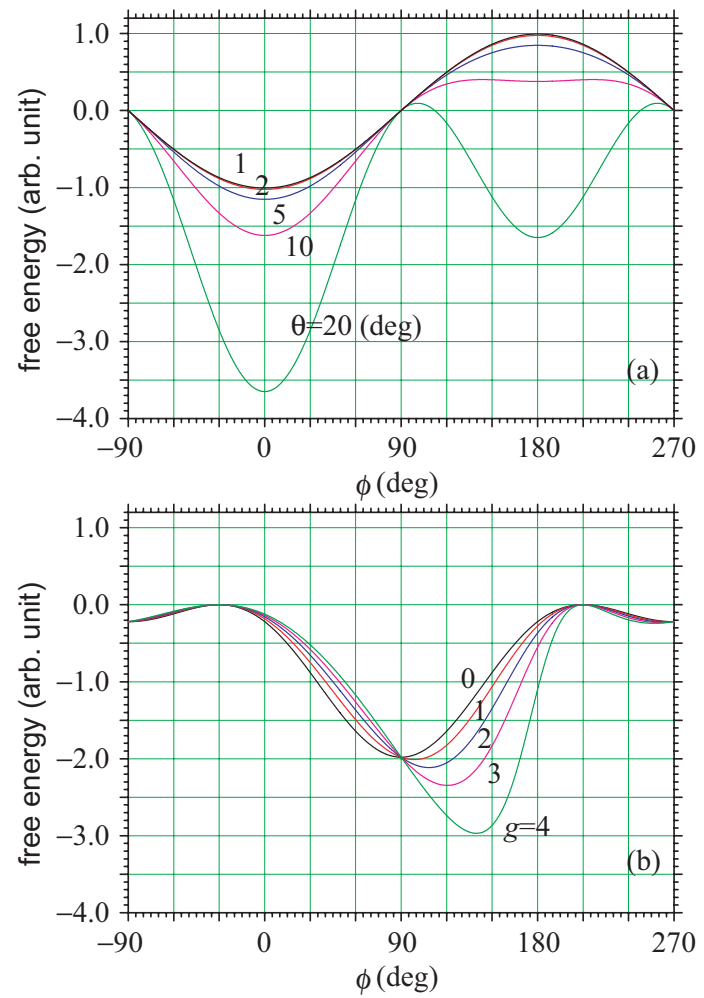

FIG. 9. (Color online) Modified free energy of Sm- $C_{\alpha}^{*}$ given in Eq. (10): (a) the polarization-independent part and (b) the polarization-dependent part. The parameters used are $a / b=0.05$, $c_{\mathrm{f}} / c_{\mathrm{p}}=-1.0$, and $\chi c_{\mathrm{p}} c_{\mathrm{f}} / a=-0.88$.

angle $\varphi$ that minimizes Eq. (10) critically depends on the shape of the polarization-independent SRILI part near $\varphi \simeq 90^{\circ}$. This SRILI part was obtained by expanding the averaged interaction potential in spherical invariants up to the lowest-order nonpolar terms containing $\cos \varphi$ and $(\cos \varphi)^{2}$ [56]. The expansion is a reasonable approximation near the minima of the SRILI part at $\varphi=0^{\circ}$ and $180^{\circ}$; apart from the minima, however, the higher-order terms containing $(\cos \varphi)^{3}$ and $(\cos \varphi)^{4}$ must play a crucial role and the shape of the SRILI part near $\varphi \simeq 90^{\circ}$ may largely be modified to reproduce the experimentally observed Sm- $C_{\alpha}^{*}$ twisting angle $\varphi$. In other words, the elastic energy for the large twist in $\mathrm{Sm}-C_{\alpha}^{*}[3,60,61]$ could not be described appropriately without considering such higher-order terms in Eq. (10). In the usual ordinary phase sequence, the importance of the higher-order terms has been alluded to in explaining the temperature dependence of the $\mathrm{Sm}-C_{\alpha}^{*}$ microscopic helix short pitch observed in several phase sequences; in particular, the similar free energy of $\mathrm{Sm}-C_{\alpha}^{*}$ without taking into account the higher-order terms always predicts the continuous change from $\mathrm{Sm}-C_{A}^{*}$ or $\mathrm{Sm}-C^{*}$ to $\mathrm{Sm}-C_{\alpha}^{*}$ and cannot explain the experimentally observed discontinuous change [8]. Appropriately treating the higher-order terms is an issue that should be addressed in the near future; it may explain not only the temperature dependence of the Sm- $C_{\alpha}^{*}$ microscopic helix short pitch but also the emergence of $\frac{3}{5}$ and $\frac{2}{3}$ in the unusual extraordinary as well as the usual ordinary phase sequences.

\section{Comparison between both models}

We now compare the predictions of these two models and consider their relative advantages and disadvantages. The phenomenological Landau model is in fact heuristic, but it has one weak point: Dolganov et al. truncated the LRILIs in Eq. (3). Direct interactions between smectic layers are short range and usually are limited to the interactions between adjacent (nearest-neighbor) layers, for positional correlations between molecules in nonadjacent layers are vanishingly small [1-3]. What is the origin of the long-range coupling between order parameters $\boldsymbol{\xi}_{i}$ in nonadjacent layers in Eqs. (2) and (3)? Note that the direct dipolar interaction between adjacent layers causes an effective long-range coupling between director orientations in different smectic layers by minimizing the free energy with respect to the polarization as actually illustrated in Eq. (11). Such an effect is rather typical for systems with several interacting thermodynamic parameters [3]. Dolganov et al. used an approximation made in Refs. [45,46] and took into account the next-nearest-neighbor and third-nearest-neighbor (TNN) interactions by cutting off the effective LRILIs beyond. The TNN interaction was necessary to stabilize $\frac{1}{3}$ in the usual ordinary phase sequence with a constant tilt angle of all the layers in the unit cell $[45,46,62,63]$. By minimizing the free energy with respect to not only the azimuthal angle $\varphi_{i}$ but also the tilt angle $\theta_{i}$, Dolganov et al. successfully stabilized $\frac{1}{4}$ and $\frac{2}{5}$ in addition to $\frac{1}{3}$ and $\frac{1}{2}$ in spite of their cutting off the effective LRILIs beyond the TNN interaction; they obtained the phase sequence of Sm- $C_{A}^{*}-\frac{1}{4}-\frac{1}{3}-\frac{2}{5}-\frac{1}{2}-\mathrm{Sm}-C^{*}$ when the frustration between $\mathrm{Sm}-C_{A}^{*}$ and $\mathrm{Sm}-C^{*}$ was explicit. It is worthwhile noticing that the neglected second flexoelectric term in Eq. (11) may play an important role in modulating $\theta_{i}$ from layer to layer within a unit cell because this flexoelectric effect produces spontaneous polarization perpendicular to the smectic plane [3].

Emelyanenko and Osipov, in contrast, carried out the free-energy minimization with respect to polarization without cutting off the effective long-range coupling. They studied the same case as Dolganov et al. did where the frustration between $\mathrm{Sm}-C_{A}^{*}$ and $\mathrm{Sm}-C^{*}$ is explicit. Since they considered that the spatially uniform tilt angle $\theta$ is rather large and temperatureindependent in this case, they naturally did not minimize the free energy with respect to the tilt angle $\theta$. By considering unit cells consisting of up to ten smectic layers [3,11], it was shown that varieties of subphases emerge between $\mathrm{Sm}-C_{A}^{*}$ and $\mathrm{Sm}-C^{*}$. The subphases appear in increasing order of $q_{T}$ and the ones with $\frac{1}{2}<q_{T}<1$ are squeezed into extremely narrow temperature ranges. In real systems, subphases with larger unit cells of irreducible $q_{T}$ in higher terms may be suppressed by such factors as surface effects, thermal fluctuations, and some other so-far neglected intermolecular interactions. Suppose subphases with $q_{T}=m / n(n \leqslant 5)$ are actually observable; their predicted phase sequence is quite similar to $\mathrm{Sm}-C_{A}^{*}-\frac{1}{5}-$ $\frac{1}{4}-\frac{1}{3}-\frac{2}{5}-\frac{1}{2}-\mathrm{Sm}-C^{*}$ from Dolganov et al. However, the subphase superlattice structure of $\frac{1}{4}$ given in Fig. 3 of Ref. [3] is profoundly different from the one shown in Fig. 8 of Ref. [35]. The difference lies not only in the modulated tilt angle within the unit cell, but also in the distorted microscopic short-pitch helical structure itself. In this way, it is intriguing to ask which model describes the nature more appropriately and to consider 
how to check this experimentally by studying the structure of the $\frac{1}{4}$ subphase in detail.

\section{ACKNOWLEDGMENTS}

The work of J.K.V. at Sungkyunkwan University was supported by World Class University program through the
National Research Foundation of Korea funded by the Ministry of Education, Science and Technology (Grant No. R32-2008000-10204-0). The visit of S.K. to Trinity College, Dublin (TCD) was funded by the SFI Walton Visiting Professorship Grant No. 11/W.1/1957. The visit of K.L.S. to TCD was facilitated through Ireland-India cooperation funds and J.K.V. thanks the Dean of FEMS for support through this award.
[1] J. Prost and R. Bruinsma, Ferroelectrics 148, 25 (1993).

[2] R. Bruinsma and J. Prost, J. Phys. (France) 4, 1209 (1994).

[3] A. V. Emelyanenko and M. A. Osipov, Phys. Rev. E 68, 051703 (2003).

[4] A. Fukuda, Y. Takanishi, T. Isozaki, K. Ishikawa, and H. Takezoe, J. Mater. Chem. 4, 997 (1994).

[5] V. P. Panov, N. M. Shtykov, A. Fukuda, J. K. Vij, Y. Suzuki, R. A. Lewis, M. Hird, and J. W. Goodby, Phys. Rev. E 69, 060701(R) (2004).

[6] N. M. Shtykov, A. D. L. Chandani, A. V. Emelyanenko, A. Fukuda, and J. K. Vij, Phys. Rev. E 71, 021711 (2005).

[7] A. D. L. Chandani, N. M. Shtykov, V. P. Panov, A. V. Emelyanenko, A. Fukuda, and J. K. Vij, Phys. Rev. E 72, 041705 (2005).

[8] K. L. Sandhya, J. K. Vij, A. Fukuda, and A. V. Emelyanenko, Liq. Cryst. 36, 1101 (2009).

[9] K. L. Sandhya, A. Fukuda, and J. K. Vij, Mol. Cryst. Liq. Cryst. 511, 36 (2009).

[10] A. D. L. Chandani, A. Fukuda, S. Kumar, and J. K. Vij, Liq. Cryst. 38, 663 (2011).

[11] K. L. Sandhya, A. D. L. Chandani, A. Fukuda, J. K. Vij, A. V. Emelyanenko, and K. Ishikawa, Phys. Rev. E 87, 012502 (2013).

[12] V. P. Panov, J. K. Vij, Y. P. Panarin, C. Blanc, V. Lorman, and J. W. Goodby, Phys. Rev. E 75, 042701 (2007).

[13] P. Mach, R. Pindak, A.-M. Levelut, P. Barois, H. T. Nguyen, C. C. Huang, and L. Furenlid, Phys. Rev. Lett. 81, 1015 (1998).

[14] P. Mach, R. Pindak, A.-M. Levelut, P. Barois, H. T. Nguyen, H. Baltes, M. Hird, K. Toyne, A. Seed, J. W. Goodby, C. C. Huang, and L. Furenlid, Phys. Rev. E 60, 6793 (1999).

[15] A.-M. Levelut and B. Pansu, Phys. Rev. E 60, 6803 (1999).

[16] T. Akizuki, K. Miyachi, Y. Takanishi, K. Ishikawa, H. Takezoe, and A. Fukuda, Jpn. J. Appl. Phys. 38, 4832 (1999).

[17] P. M. Johnson, D. A. Olson, S. Pankratz, T. Nguyen, J. Goodby, M. Hird, and C. C. Huang, Phys. Rev. Lett. 84, 4870 (2000).

[18] A. Cady, J. A. Pitney, R. Pindak, L. S. Matkin, S. J. Watson, H. F. Gleeson, P. Cluzeau, P. Barois, A.-M. Levelut, W. Caliebe, J. W. Goodby, M. Hird, and C. C. Huang, Phys. Rev. E 64, 050702(R) (2001).

[19] I. Musevic and M. Skarabot, Phys. Rev. E 64, 051706 (2001).

[20] P. Fernandes, P. Barois, E. Grelet, F. Nallet, J. W. Goodby, M. Hird, and J.-S. Micha, Eur. Phys. J. E 20, 81 (2006).

[21] S. Jaradat, P. D. Brimicombe, C. Southern, S. D. Siemianowski, E. DiMasi, M. Osipov, R. Pindak, and H. F. Gleeson, Phys. Rev. E 77, 010701(R) (2008).

[22] S. Wang, L. D. Pan, R. Pindak, Z. Q. Liu, H. T. Nguyen, and C. C. Huang, Phys. Rev. Lett. 104, 027801 (2010).

[23] M. Fukui, H. Orihara, Y. Yamada, N. Yamamoto, and Y. Ishibashi, Jpn. J. Appl. Phys. 28, L849 (1989).
[24] A. D. L. Chandani, Y. Ouchi, H. Takezoe, A. Fukuda, K. Terashima, K. Furukawa, and A. Kishi, Jpn. J. Appl. Phys. 28, L1261 (1989).

[25] A. D. L. Chandani, E. Gorecka, Y. Ouchi, H. Takezoe, and A. Fukuda, Jpn. J. Appl. Phys. 28, L1265 (1989).

[26] V. Laux, N. Isaert, H. T. Nguyen, P. Cluzeau, and C. Destrade, Ferroelectrics 179, 25 (1996).

[27] D. Schlauf, C. Bahr, and H. T. Nguyen, Phys. Rev. E 60, 6816 (1999).

[28] P. M. Johnson, S. Pankratz, P. Mach, H. T. Nguyen, and C. C. Huang, Phys. Rev. Lett. 83, 4073 (1999).

[29] S. T. Wang, Z. Q. Liu, B. K. McCoy, R. Pindak, W. Caliebe, H. T. Nguyen, and C. C. Huang, Phys. Rev. Lett. 96, 097801 (2006).

[30] K. L. Sandhya, Y. P. Panarin, U. Manna, J. K. Vij, and S. Kumar, Phys. Rev. Lett. 98, 219801 (2007).

[31] K. L. Sandhya, J. K. Song, Y. P. Panarin, J. K. Vij, and S. Kumar, Phys. Rev. E 77, 051707 (2008).

[32] B. K. McCoy, Z. Q. Liu, S. T. Wang, L. Pan, S. Wang, H. T. Nguyen, R. Pindak, and C. C. Huang, Phys. Rev. E 77, 061704 (2008).

[33] S. Wang, L. D. Pan, B. K. McCoy, S. T. Wang, R. Pindak, H. T. Nguyen, and C. C. Huang, Phys. Rev. E 79, 021706 (2009).

[34] P. V. Dolganov, V. M. Zhilin, V. K. Dolganov, and E. I. Kats, Phys. Rev. E 86, 020701(R) (2012).

[35] P. V. Dolganov, V. M. Zhilin, V. K. Dolganov, and E. I. Kats, Phys. Rev. E 83, 061705 (2011).

[36] P. V. Dolganov, V. M. Zhilin, V. K. Dolganov, and E. I. Kats, Phys. Rev. E 82, 040701 (2010).

[37] P. V. Dolganov, V. M. Zhilin, V. K. Dolganov, and E. I. Kats, Phys. Rev. E 67, 041716 (2003).

[38] H. Orihara, Y. Naruse, M. Yagyu, A. Fajar, and S. Uto, Phys. Rev. E 72, 040701(R) (2005).

[39] M. Johno, K. Itoh, J. Lee, Y. Ouchi, H. Takezoe, A. Fukuda, and T. Kitazume, Jpn. J. Appl. Phys. 29, L107 (1990).

[40] K. Hiraoka, H. Takezoe, and A. Fukuda, Ferroelectrics 147, 13 (1993).

[41] T. Qian and P. L. Taylor, Phys. Rev. E 60, 2978 (1999).

[42] L. A. Parry-Jones and S. J. Elston, Phys. Rev. E 63, 050701(R) (2001)

[43] L. D. Pan, P. Barois, R. Pindak, Z. Q. Liu, B. K. McCoy, and C. C. Huang, Phys. Rev. Lett. 108, 037801 (2012).

[44] A. V. Emelyanenko and M. A. Osipov, Ferroelectrics 309, 13 (2004)

[45] M. Cepic and B. Zeks, Phys. Rev. Lett. 87, 085501 (2001).

[46] M. Cepic, E. Gorecka, D. Pociecha, B. Zeks, and H. T. Nguyen, J. Chem. Phys. 117, 1817 (2002).

[47] M. Cepic and B. Zeks, Mol. Cryst. Liq. Cryst. 263, 61 (1995).

[48] A. Roy and N. V. Madhusudana, Eur. Phys. J. E 1, 319 (2000). 
[49] K. Miyachi, M. Kabe, K. Ishikawa, H. Takezoe, and A. Fukuda, Ferroelectrics 147, 147 (1993).

[50] M. A. Osipov and M. V. Gorkunov, Liq. Cryst. 33, 1133 (2006).

[51] N. M. Shtykov, J. K. Vij, R. A. Lewis, M. Hird, and J. W. Goodby, Phys. Rev. E 62, 2279 (2000).

[52] M. Torikai and M. Yamashita, Mol. Cryst. Liq. Cryst. 465, 239 (2007).

[53] Y. Sugimoto, M. Torikai, and M. Yamashita, Mol. Cryst. Liq. Cryst. 511, 348 (2009).

[54] Y. Sakai, M. Torikai, and M. Yamashita, Mol. Cryst. Liq. Cryst. 516, 81 (2010).

[55] C. S. O. Yokoi, L.-H. Tang, and W. Chou, Phys. Rev. B 37, 2173 (1988).
[56] M. A. Osipov and A. Fukuda, Phys. Rev. E 62, 3724 (2000).

[57] A. Fukuda, H. Hakoi, M. Sato, and M. A. Osipov, Mol. Cryst. Liq. Cryst. 398, 169 (2003).

[58] Y. Takanishi, K. Hiraoka, V. K. Agrawal, H. Takezoe, A. Fukuda, and M. Matsushita, Jpn. J. Appl. Phys. 30, 2023 (1991).

[59] T. Isozaki, K. Hiraoka, Y. Takanishi, H. Takezoe, A. Fukuda, Y. Suzuki, and I. Kawamura, Liq. Cryst. 12, 59 (1992).

[60] M. Skarabot, M. Cepic, B. Zeks, R. Blinc, G. Heppke, A. V. Kityk, and I. Musevic, Phys. Rev. E 58, 575 (1998).

[61] T. Y. Tan, L. H. Ong, and M. Cepic, Mol. Cryst. Liq. Cryst. 540, 69 (2011).

[62] Y. Yamada and N. Hamaya, J. Phys. Soc. Jpn. 52, 3466 (1983).

[63] T. Matsumoto, A. Fukuda, M. Johno, Y. Motoyama, T. Yui, S.-S. Seomun, and M. Yamashita, J. Mater. Chem. 9, 2051 (1999). 